\title{
Real Life Data for Glecaprevir/Pibrentasvir: A Single-Center Study
}

\author{
Fatma Çölkesen $^{1}$ iD $\quad$ Arzu Tarakçı ${ }^{1}$ iD $\quad$ Fatma Kacar $^{1}$ (D) $\quad$ Esma Eroğlu $^{1}$ \\ Şule Özdemir Armağan ${ }^{1}$ (I)
}

1 Health Sciences University, Konya Training and Research Hospital, Department of Infectious Diseases and Clinical Microbiology, Konya, Turkey

\begin{abstract}
Background: Licensing of next-generation direct-acting antiviral agents (DAA) has revolutionized the treatment of Hepatitis C Virus (HCV) infection. These agents are important due to their high virological efficacy, high resistance barrier, short duration of treatment, and rare serious side effects. The purpose of our study was to present real-life data of chronic hepatitis $\mathrm{C}$ (CHC) infected patients with genotypes 2 and 3 who were treated with glecaprevir/pibrentasvir regimen.

Methods: Glecaprevir/pibrentasvir treatment was initiated in 127 patients infected with CHC genotype 2 and 3 . Patients received glecaprevir/pibrentasvir (100 mg/40 mg) orally in the form of three tablets once a day as per recommendations of the Republic of Turkey, Ministry of Health. In the assessments of patients at the time of diagnosis and following DAA use, virological response criteria HCV-RNA value was $<25 \mathrm{IU} / \mathrm{ml}$.
\end{abstract}

Results: 127 patients were included in the study. The mean age of the patients was $27 \pm 6$, and 125 patients (98.4\%) were male. 118 patients $(92.9 \%)$ had a history of intravenous drug use, and 78 patients $(61.4 \%)$ were convicts. $83.6 \%$ (n:106) of the patients were genotype 3, 15\% (n:19) genotype 2, $0.7 \%$ (n:1) genotype $1+3$, and $0.7 \%$ (n:1) genotype $3+4$. The mean liver fibrosis stage was $1.7 \pm 0.8$ and histological activity index was 7.9 $2.7 .99 .2 \%$ (n:126) of the patients achieved virological response and sustained virological response (SVR)-12, and only 1 patient did not achieve end-of-treatment response and SVR-12.

Conclusion: An effective, well-tolerated, oral and short-term treatment for patients infected with CHC genotypes 2 and 3 is currently possible with glecaprevir/pibrentasvir.

Key words: Chronic Hepatitis C, Direct-Acting Antiviral Agent, Glecaprevir, Pibrentasvir.

\section{INTRODUCTION}

Chronic hepatitis $\mathrm{C}(\mathrm{CHC})$ infection is one of the main causes of chronic liver diseases across the world. Liver damage may vary from minimal histological changes to severe fibrosis, cirrhosis and hepatocellular carcinoma (HCC). Approximately 71 million people are infected with CHC infection worldwide $(1,2)$. In the studies conducted on blood donors in Turkey, HCV prevalence was 0.3$1.8 \%$. It is estimated that there are $350000-700000$ patients infected with Hepatitis C Virus (HCV) in Turkey (3). All patients with $\mathrm{CHC}$ are potential candidates for antiviral therapy (4). The primary objective in the treatment of $\mathrm{CHC}$ is to achieve sustained virological response (SVR), which is also expressed as a cure, and to prevent mortality and morbidity due to complications (5). 
The first treatment in CHC started with standard alpha interferon (IFN) monotherapy in 1991, and with the addition of ribavirin (RBV) to this monotherapy in 1997, SVR rates increased from $6 \%$ to $34 \%$ in 24 weeks of treatment, and from $16 \%$ to $42 \%$ in 48 weeks of treatment (6). In 2001, it was attempted to extend the half-life with pegylation of IFN in order to increase virological response rates. Even though 48 weeks of treatment with Peg-IFN+RBV, SVR rates increased to $56 \%$, adequate treatment rate was not reached and side effects rates were not reduced (7). Over time, HCV's life cycle and pathogenesis were better understood, and treatments that directly targeted the virus were developed. Licensing of next-generation direct-acting antiviral agents (DAA) has revolutionized the treatment of $\mathrm{HCV}$ infection. These agents are important due to their high virological efficacy, high resistance barrier, short duration of treatment and rare serious side effects (8).

Glecaprevir/pibrentasvir (MAVIRET TM) is a fixed-dose combination tablet of glecaprevir, a NS3/4A prosthesis inhibitor, and pibrentasvir, a NS5A inhibitor, and has been developed by Abbvie for the treatment of $\mathrm{CHC}$ (9). Glecaprevir/pibrentasvir was formulated as a single tablet at a fixed dose (100 mg/40 mg, respectively). The recommended daily dose is three tablets taken orally once a day including $300 \mathrm{mg}$ of glecaprevir and 120 $\mathrm{mg}$ of pibrentasvir. This fixed-dose oral combination of glecaprevir/pibrentasvir was approved by the Food and Drug Administration (FDA) in August 2017 in noncirrhotic or compensated cirrhotic genotype 1, 2, 3, 4, 5 or 6 CHC patients. In March 2019, it was included into the reimbursement plan within the scope of Health Implementation Communique. The purpose of our study was to present real-life data of CHC-infected patients with genotypes 2 and 3 who were treated with glecaprevir/ pibrentasvir regimen.

\section{MATERIALS AND METHODS}

This study was approved by the local ethics committee of University of Health Sciences, Konya Training and Research Hospital (Date: 08.03.2020, No:36-15), and the study was conducted according to the Declaration of Helsinki 1975.

\section{Study Design}

Between March 2019 and January 2020, patients who were followed up with the diagnosis of $\mathrm{CHC}$ at the infectious diseases and clinical microbiology clinic of the Health Sciences University of Konya Training and Research
Hospital were included in the study. The inclusion criteria were: 1 - Patients who were $>18$ years old; 2 - Patients whose HCV-RNA values have been detectable for $\geq 6$ months (CHC diagnostic criteria); 3- Patients who were infected with $\mathrm{CHC}$ genotype 2 or 3.137 patients who met these criteria were included in the study. 10 patients who did not complete the 8-week glecaprevir/pibrentasvir treatment were excluded from the study.

\section{Data Collection}

Clinical, demographic and laboratory data of the patients were retrieved from the hospital database. Liver biopsies were performed percutaneously and evaluated based on the Ishak scoring system. According to this system, 0-2 points were classified as "no fibrosis or minimal fibrosis", 3 points as "portal fibrosis", 4 points as "bridging fibrosis" and 5-6 points as "cirrhosis".

\section{Laboratory Tests}

Real-time polymerase chain reaction (Anatolia Geneworks HCV, Turkey) was used to detect HCV RNA and HCV genotype. Hemogram analysis was performed with Sysmex XN-1000 (Sysmex, Kobe, Japan). HCV RNA measurements and other tests were performed at weeks 0, 4, 8 and 12 .

\section{Management of Glecaprevir/Pibrentasvir Treatment in Patients with Chronic Hepatitis C}

The administered treatment and the duration of treatment was determined according to the National Healthcare Practice Communique of the Republic of Turkey Ministry of Health. Based on this guideline, glecaprevir/pibrentasvir is used for a total of 8 weeks in treatment-naive, non-cirrhotic patients with CHC genotype 2 and 3 and for a total of 12 weeks for cirrhotic patients. For non-cirrhotic patients with $\mathrm{CHC}$ genotype 2 who have received prior treatment, the duration of therapy with glecaprevir/pibrentasvir for NS5A-inhibitor-naive patients is 8 weeks in total, and it is 16 weeks in total for patients who have received a NS5A inhibitor previously. The duration of treatment with glecaprevir/pibrentasvir in non-cirrhotic patients with genotype 3 who have received any prior treatment is 16 weeks, while it is 16 weeks in total for cirrhotic (Child-Pugh A) patients. Patients received glecaprevir/pibrentasvir (100 mg/40 mg) (Maviret; Abbvie) orally in the form of three tablets once a day as per the recommendations of the Republic of Turkey Ministry of Health. In the assessment of the data of patients at the time of diagnosis and following DAA use, $<25 \mathrm{IU} / \mathrm{ml} \mathrm{HCV-RNA} \mathrm{value} \mathrm{was} \mathrm{accepted} \mathrm{as}$ virological response criteria. 


\section{Ethical Approval}

This study was approved by the local ethics committee of University of Health Sciences, Konya Training and Research Hospital, with the 08/03/2020/36-15 ID number, and the study was conducted according to the Declaration of Helsinki 1975.

\section{Statistical Analysis}

SPSS version 20.0 (IBM SPSS Statistics 20.0) was used for data evaluation and analysis. Continuous variables were expressed as median (minimum-maximum) and mean \pm standard deviation values. Categorical variables were expressed as n $(\%)$.

Table 1. Demographic Data of Patients

\begin{tabular}{|l|c|}
\hline Gender & $\%$ (n) \\
\hline Female & $1.6 \%(2 / 127)$ \\
\hline Male & $98.4 \%(125 / 127)$ \\
\hline Age & $27 \pm 6(19-69)$ \\
\hline Treatment History & \\
\hline Treatment-Naive & $96.9 \%(123 / 127)$ \\
\hline Received Prior Treatment & $3.1 \%(4 / 127)$ \\
\hline Genotype Distribution & \\
\hline Genotype 3 & $83.6 \%(106 / 127)$ \\
\hline Genotype 2 & $15 \%(19 / 127)$ \\
\hline Genotype 1+3 & $0.7 \%(1 / 127)$ \\
\hline Genotype 3+4 & $0.7 \%(1 / 127)$ \\
\hline
\end{tabular}

Table 2. Laboratory Parameters Prior to Treatment of Patients

\begin{tabular}{|l|c|}
\hline HCV RNA & $\begin{array}{c}3149060 \mathrm{IU} / \mathrm{ml}(1333- \\
58490000 \mathrm{IU} / \mathrm{ml})\end{array}$ \\
\hline Fibrosis stage & $1,7 \pm 0,8$ \\
\hline Histological Activity Index & $7,9 \pm 2,7$ \\
\hline ALT & $93.5 \mathrm{u} / \mathrm{L}(10-591 \mathrm{u} / \mathrm{L})$ \\
\hline AST & $53.2 \mathrm{u} / \mathrm{L}(16-289 \mathrm{u} / \mathrm{L})$ \\
\hline
\end{tabular}

\section{RESULTS}

127 patients who completed the eight weeks of treatment were included in the study. The mean age of the patients was $27 \pm 6$, and 125 (98.4\%) were male. Four $(3.1 \%)$ patients received prior treatment, and 123 (96.9\%) patients were treatment-naive. Three patients who received prior treatment had relapsed after one year of peg-IFN+ribavirin treatment, while one patient discontinued sofosbuvir therapy. All patients were noncirrhotic. Four patients had asthma, one had chronic renal failure (CRF), and one had hypogonadotropic hypogonadism. 118 (92.9\%) patients had a history of intravenous drug use. $78(61.4 \%)$ patients were convicts. Genotype distributions were as follows: $83.6 \%$ (n:106) of the patients were genotype $3,15 \%$ (n:19) were genotype 2 , $0.7 \%$ (n:1) was genotype $1+3,0.7 \%$ (n:1) was genotype $3+4$. $28(20.4 \%)$ patients had a liver biopsy before treatment was initiated. The mean stage of fibrosis was $1.7 \pm 0.8$, and the mean histological activity index (HAI) was 7.9 \pm 2.7 . Patients who completed 8 weeks of treatment achieved a virological response; however, only one patient did not achieve end-of-treatment response. All 126 patients who achieved a treatment response and completed a 12week follow-up achieved SVR-12; however, SVR-12 rate was $99.2 \%$ due to the one patient who did not develop a response at the end of treatment. As adverse events, four patients had weakness, one patient had change in taste sensation, one patient had diarrhea, one patient had nosebleed, one patient had bleeding in gums, one patient had constipation and one patient had stomach complaints. All complaints occurred in the first weeks of treatment and resolved spontaneously in the next weeks. There were no serious adverse events that resulted in interruption of treatment or discontinuation of treatment.

\section{DISCUSSION}

Glecaprevir/pibrentasvir has shown great improvement in real-life data regardless of baseline patient characteristics such as gender, age, fibrosis stage, kidney function and past HCC therapy (10). In our study, SVR12 rate was $99.2 \%$ in genotype 2 and 3 patients, only one patient did not achieve end-of-treatment response and SVR-12. This patient was a convicted patient infected with genotype 3 and had no other known chronic disease. $\mathrm{HCV}$ RNA result was negative at the end of the fourth week; however, at the end of the eighth week, it was 
positive again. It is believed that the patient did not use the drug or used it irregularly; however, the patient could not be reached again, as the patient was a convict and the pandemic had started. The efficacy of glecaprevir/ pibrentasvir in non-cirrhotic or compensated cirrhotic patients infected with CHC genotype 3 was investigated in studies ENDURANCE-3 and SURVEYOR-2 (Section 3). ENDURANCE-3 is a partially randomized, actively controlled and open-label study in treatment-naive patients. In this study, SVR-12 rates were 94.9\% (149/157) and 95.3\% (222/233), respectively in 8-week and 12-week glecaprevir/pibrentasvir treatment groups (11). SURVEYOR-2 is an open-label study in which 12 or 16 weeks of glecaprevir/pibrentasvir treatment was randomized in patients with non-cirrhotic or compensated cirrhosis. In this study, SVR-12 rate was 98\% (39/40) with 12-week treatment in treatment-naive cirrhotic patients, and SVR-12 rate was 96\% (66/99) with 16-week treatment in cirrhotic patients who received prior treatment (12). In the study which presented reallife data of patients with genotype 1-4 CHC in Italy, SVR rates were $99.2 \%$ and $100 \%$, respectively, for 8 weeks and 12-16 weeks of glecaprevir/pibrentasvir treatment (13).

There are studies that show that glecaprevir/pibrentasvir is also effective in specific patient populations. EXPEDITION-4 is a single-arm and open-label study. In this study, SVR rate was $98 \%$ in non-cirrhotic and compensated cirrhotic patients with chronic renal failure (CRF) stage 4 and 5 (82\% was undergoing hemodialysis) and genotype 1-6 infection. It has been emphasized that renal failure does not affect the effectiveness of glecaprevir/pibrentasvir and that dose adjustment is not required in these patients (14). MAGELLAN-2 is an openlabel, single-arm study that evaluated patients with liver and kidney transplants and CHC genotype 1-6 infection. Patients were receiving cyclosporine $\leq 100 \mathrm{mg}$, sirolimus, tacrolimus, everolimus, mycophenolate, azathioprine and prednisolone, and the SVR-12 rate was 98\% (15). In a study conducted in non-cirrhotic patients with $\mathrm{CHC}$, genotype 1-6 co-infected with human immunodeficiency virus (HIV), SVR-12 rate was $98 \%$ with glecaprevir/ pibrentasvir treatment. Glecaprevir/pibrentasvir was also found to be effective in HIV-infected patients (16).

Severe side effects were observed with telaprevir, another DAA, which was previously used. These side effects led to a treatment discontinuation rate of $8-12 \%$ $(17,18)$. In several studies, very few side effects associated with glecaprevir/pibrentasvir were reported $(13,19)$. The most common complaints in our study were weakness, gastrointestinal tract complaints, nose bleeds and bleeding in gums. However, these side effects were not very severe and occurred at the beginning of treatment and then resolved. No patient discontinued treatment due to side effects. In a combined analysis of tolerability data of 8,12 and 16 weeks of treatment with glecaprevir/pibrentasvir, the most commonly reported side effects were headache $(13 \%)$, fatigue $(11 \%)$ and nausea $(8 \%)$ (20). In another study, there was a high discontinuation rate in treatment with glecaprevir/pibrentasvir among patients over the age of 75 years, and it was recommended that adequate care be provided for elderly patients (10).

This study has some limitations. Most of the patients included in the study were convicts, which reduced treatment compliance, and there were patients who discontinued the treatment. Moreover, this was a descriptive study, and there was no comparison between the subgroups. The main reason for the inability to compare is that the distribution of subgroups were uneven. Another limitation is that the cause of the lack of response in one patient was not clarified as the only patient who was not able to receive an end-of-treatment response and SVR did not attend the follow-up.

In conclusion, there are currently effective, well tolerated, oral and interferon-free treatment regimens involving direct-acting antiviral agents for almost all patients infected with genotypes 2 and 3. In this study, our treatment experience with the glecaprevir/pibrentasvir regimen is presented. It has been determined that this regime is safe, well tolerated and has high efficiency in the treatment of $\mathrm{CHC}$.

\section{Declarations}

The authors received no financial sport for the research and/or authorship of this article. There is no conflict of interest.

This study was approved by the local ethics committee of University of Health Sciences, Konya Training and Research Hospital (Date: 08.03.2020, No:36-15), and the study was conducted according to the Declaration of Helsinki 1975. 


\section{REFERENCES}

1. Polaris Observatory HCV Collaborators. Global prevalence and genotype distribution of hepatitis $C$ virus infection in 2015: a modeling study. Lancet Gastroenterol Hepatol. 2017;2:161-176.

2. European Union HCV Collaborators. Hepatitis C virus prevalence and level of intervention required to achieve the WHO targets for elimination in the European Union by 2030: a modeling study. Lancet Gastroenterol Hepatol. 2017;2:325-336.

3. Tozun N, Ozdogan O, Cakaloglu Y, Idilman R, Karasu Z, Akarca U et al. Seroprevalence of hepatitis $\mathrm{B}$ and $\mathrm{C}$ virus infections and risk factors in Turkey: a fieldwork TURHEP study. Clin Microbiol Infect. 2015;21(11):1020-6.

4. Pawlotsky JM, Negro F, Aghemo A, Berenguer M, Dalgard O, Dusheiko G et al. EASL Recommendations on Treatment of Hepatitis C 2018. J Hepatol. 2018;69(2):461-511.

5. Van der Meer AJ, Veldt BJ, Feld JJ, Wedemeyer H, Dufour JF, Lammert $\mathrm{F}$ et al. Association between sustained virological response and allcause mortality among patients with chronic hepatitis $\mathrm{C}$ and advanced hepatic fibrosis. Jama. 2012;308(24):2584-2593.

6. Linas BP, Wong AY, Schackman BR, Kim AY, Freedberg KA. Cost effective screening for acute hepatitis $C$ virus infection in hiv-infected men who have sex with men. Clin Infect Dis. 2012;55(2):279-90.

7. Hadziyannis SJ, Sette H, Jr, Morgan TR, et al. Peginterferon alfa-2a and ribavirin combination therapy in chronic hepatitis C: A randomized study of treatment duration and ribavirin dose. Ann Intern Med. 2004;140:346-55.

8. Carrion AF, Martin P. Glecaprevir + pibrentasvir for treatment of hepatitis C. Expert Opin Pharmacother. 2018;19(4):413-419.

9. Poordad F, Felizarta F, Asatryan A, Sulkowski MS, Reindollar RW, Landis CS et al. Glecaprevir and pibrentasvir for 12 weeks for hepatitis $C$ virus genotype 1 infection and prior direct-acting antiviral treatment. Hepatology. 2017;66(2):389-397.

10. Tamori A,Inoue K, Kagawa T, Takaguchi K, Nouso K, Iwasaki $Y$ et al. Intention to treat assessment of glecaprevir + pibrentasvir combination therapy for patients with chronic hepatitis $\mathrm{C}$ in the real world. Hepatology Res. 2017;49(12), 1365-1373.

11. Foster GR,Gane E, Asatryan A, Asselah T, Ruane PJ, Pol S et al. ENDURANCE-3: safety and efficacy of glecaprevir/pibrentasvir compared to sofosbuvir plus daclatasvir in treatment-naïve HCV genotype 3-infected patients without cirrhosis. J Hepatol. 2017; 66(1), S33.

12. Wyles D, Poordad F, Wang S, Alric L, Felizarta F, Kwo PY et al.Glecaprevir/Pibrentasvir for HCV Genotype 3 Patients with Cirrhosis and/or Prior Treatment Experience: A Partially Randomized Phase III Clinical Trial. Hepatology. 2018;67(2):514-523.

13. D'Ambrosio R,Pasulo L, Puoti M, Vinci M, Schiavini M, Lazzaroni S et al. Real-world effectiveness and safety of glecaprevir/pibrentasvir in 723 patients with chronic hepatitis C. J Hepatol. 2019, 70(3), 379-387.

14. Gane E, Lawitz E, Pugatch D, Papatheodoridis G, Bräu N, Brown A et al. Glecaprevir and pibrentasvir in patients with $\mathrm{HCV}$ and severe renal impairment. N Engl J Med. 2017;377:1448-1455.
15. Asselah T, Lee SS, Yao BB, Nguyen T, Wong F, Mahomed A et al. Efficacy and safety of glecaprevir/ pibrentasvir in patients with chronic hepatitis $C$ virus genotype 5 or 6 infection (ENDURANCE-5,6): an open-label, multicentre, phase $3 \mathrm{~b}$ trial. Lancet Gastroenterol Hepatol. 2019;4:45-51.

16. Rockstroh JK,Lacombe K, Viani RM, Orkin C, Wyles D, Luetkemeyer AF et al. Efficacy and safety of glecaprevir/pibrentasvir in patients coinfected with hepatitis $C$ virus and human immune deficiency virus type 1: the EXPEDITION-2 study. Clin Infect Dis. 2018;67(7), 10101017.

17. Jacobson IM, McHutchison JG, Dusheiko G, Di Bisceglie AM, Reddy KR, Bzowej NH et al; ADVANCE Study Team. Telaprevir for previously untreated chronic hepatitis $C$ virus infection. N Engl J Med. 2011;364:2405-16.

18. Zeuzem S, Andreone P, Pol S, Lawitz E, Diago M, Roberts S et al; REALIZE Study Team. Telaprevir for retreatment of HCV infection. N Engl J Med. 2011;364:2417-28.

19. Osawa M, Imamura M, Teraoka Y, Uchida T, Morio K, Fujino H et al. Real-world efficacy of glecaprevir plus pibrentasvir for chronic hepatitis C patient with previous direct-acting antiviral therapy failures. J Gastroenterol. 2019; 54: 291-6.

20. Dufour JF, Zuckerman E, Zadeikis N, Hezode C, Paik SW, Andreone $\mathrm{P}$ et al. Safety of glecaprevir/ pibrentasvir in adults with chronic genotype 1-6 hepatitis C virus infection: an integrated analysis [abstractno. FRI-238]. J Hepatol. 2017;66(1 Suppl):S515. 